Dig Surg 2007;24:68-69

DOI: $10.1159 / 000100922$

\section{Parotid Metastasis from a Gastroesophageal Carcinoma: Report of a Case}

\author{
J.M. Schoneveld ${ }^{a}$, W.L.E.M. Hesp ${ }^{a}$, T.M. Teune ${ }^{b}$ \\ ${ }^{a}$ Department of Surgery, Albert Schweitzer Hospital, and \\ b Laboratory for Pathology, Dordrecht, The Netherlands
}

\section{Key Words}

Gastroesophageal carcinoma $\cdot$ Metastasis $\cdot$ Parotid gland

\section{Abstract}

Carcinomas of the lower esophagus, gastroesophageal junction or stomach rarely metastasize to the cervical lymph nodes. Furthermore, the parotid gland is an even more unusual site of metastasis from a carcinoma located at these sites. We describe the case of a 45-year-old male patient who was diagnosed 2 months after transhiatal gastroesophagectomy for a primary gastric adenocarcinoma with metastasis in the left parotid gland. In the literature we have only found one other case report. C Copyright $\odot 2007$ S. Karger AG, Base

\section{Introduction}

Metastatic tumors to the parotid gland are very uncommon and account for $8 \%$ of all malignancies of the parotid gland. The parotid gland and its lymph nodes are possible sites of metastasis from head and neck cancers, especially squamous cell carcinoma or melanoma [1]. Metastases from below the clavicle are mostly from renal and lung cancers but adenocarcinoma originating from the breast [2], prostate [3] and colon [4] have also been reported to metastasize to the parotid gland. We report the metastasis of an adenocarcinoma of the gastroesophageal junction and proximal stomach to the parotid gland. In the literature only one recent case has been reported so far [5].

\section{Case Report}

A 45-year-old man was referred with progressive dysphagia of 2 months duration and a significant weight loss of $12 \mathrm{~kg}$. He reported no other health problems or specific gastrointestinal complaints, including gastric reflux. An upper gastrointestinal endoscopy was performed, which revealed a suspect, exophytic, growing mass at the gastroesophageal junction and proximal stomach. Transesophageal ultrasonography consequently showed a tumor $40-50 \mathrm{~cm}$ from the dental row with evidence of invasion of the muscular gastric wall as well as the presence of 6 pathologically enlarged lymph nodes (diameters maximizing from 1 to $2 \mathrm{~cm}$ ). Contrast-enhanced computed tomography of thorax and abdomen showed no evidence of distal metastases. Ultrasonography of the supraclavicular region and neck revealed no pathologically enlarged lymph nodes.

Biopsies from the gastric mass, obtained during endoscopy, rendered the diagnosis of an adenocarcinoma. Consequently, the patient underwent a transhiatal esophagectomy with a gastric tube pull-up and cervical esophagogastric anastomosis. Intraoperatively, splenectomy was necessitated because of adhesions between the stomach and spleen. Postoperative histopathological examination of the specimen confirmed the initial diagnosis of an 8-cm diameter adenocarcinoma arising in the proximal stomach and extending to the gastroesophageal junction (fig. 1). The tumor was found to grow through the gastric muscular wall into the surrounding serosal fat, as had been suspected from the results of the imaging techniques. All 14 lymph nodes resected were free of metastases. No extension of the tumor into the spleen was found. Based on these findings, the oncology advisory review team issued clinical follow-up.

Two months after surgery, the patient returned to our clinic with a rapidly growing mass in the left preauricular region. A new computed tomography of the thorax and abdomen showed no lymphadenopathies or signs of early metastasis of his gastric carcinoma. Fine-needle aspiration of the preauricular lesion showed atypical cells, but was inconclusive for malignancy. A superficial parotidectomy was performed. A 4-cm diameter tumor was excised, preserving the facial nerves and its branches. Intraoperative frozen section examination was inconclusive for malignancy. However, histopathological examination of the final routinely $\mathrm{HE}$ and immunohistochemically stained slides, confirmed later on by pathology peer review, showed that the preauricular tumor indeed was a metastasis of his previously diagnosed gastric carcinoma (fig. 2). The patient was first treated with chemotherapy using taxol and carboplatin. Secondary local radiotherapy was started for the preauricular region.

Nine months after the initial operation an MRI of the brain was made because of a newly diagnosed epileptic insult. No signs of brain metastases were seen.

\section{Discussion}

The most common sites of metastases in patients with gastric cancer are the liver, peritoneum, omentum, lungs and mesentery. Local invasion, lymphatic and hematogenous metastases are the major modes of spreading gastric cancer.

In our case the tumor was found to grow through the gastric muscular wall into the surrounding serosal fat but no further local invasion was seen. Lymphatic metastases follow the route of lymph drainage to the perigastric region, or sometimes distant metastases occur in the supraclavicular lymph nodes via the thoracic duct [6]. The supraclavicular region is not drained by the afferents of the parotid gland, therefore a lymphatic route does not seem likely in this case. Moreover, in our case, postoperative histopathological findings showed no evidence of lymph node metastases.

The third way of tumor spread is the hematogenous route where metastatic emboli travel the vascular system, becoming entrapped in regions of vascular medullar tissue and in regions of slowed circulation [7]. The metastatic emboli may travel the portal system to the liver and further on to the lungs. Metastases may also occur directly in the head and neck by the paravertebral plexus (Batson plexus), bypassing the portal vein and the filtering system of the lungs [8]. This alternate route is described in two similar cases where a gastric adenocarcinoma metastasized to the parotid gland [8] and an esophageal carcinoma metastasized to 


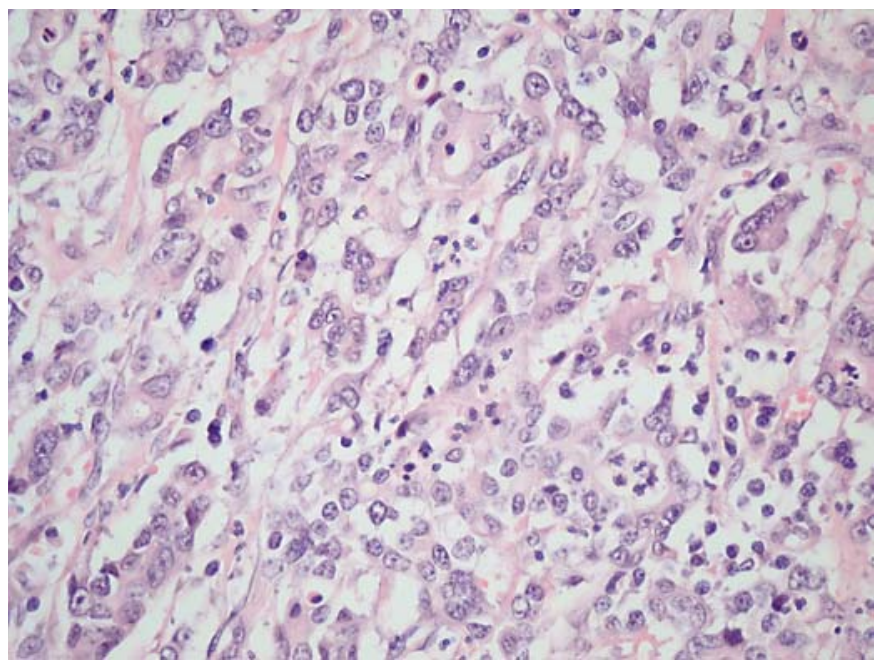

Fig. 1. Gastroesophageal carcinoma. $\times 400$. HE.

the jaw [9]. Despite Breschet's detailed description of the vertebral venous system in 1819, it was only in 1940 that Batson [8] demonstrated the preponderant role it plays in the propagation of metastatic carcinomatosis, infection or gas emboli.

To our knowledge, a solitary distant metastasis of a gastric carcinoma in such a short time and without other tumor spread has not been described before. The previous case report mentioned other distant metastases shortly after or concomitant with the diagnosis of gastric carcinoma, and the parotid metastasis occurred several years after surgery [5]. In our case, both preoperatively and 2 months after surgery, no signs of nearby or distant metastases could be demonstrated with imaging techniques, or in the surgical specimen. Therefore, it was concluded that the tumor had most likely spread through an alternate hematogenous route by way of the Batson plexus, which is postulated as a direct path to the head and neck region whereby the liver and lungs are bypassed.

\section{References}

1 Batsakis JG: Pathology consultation. Parotid gland and its lymph nodes as metastatic sites. Ann Otol Rhinol Laryngol 1983; 92: 209-210.

- 2 Bissett D, Bessell EM, Bradley PJ, Morgan DA, McKenzie CG: Parotid metastasis from carcinoma of the breast. Clin Radiol 1989;40:309310 .

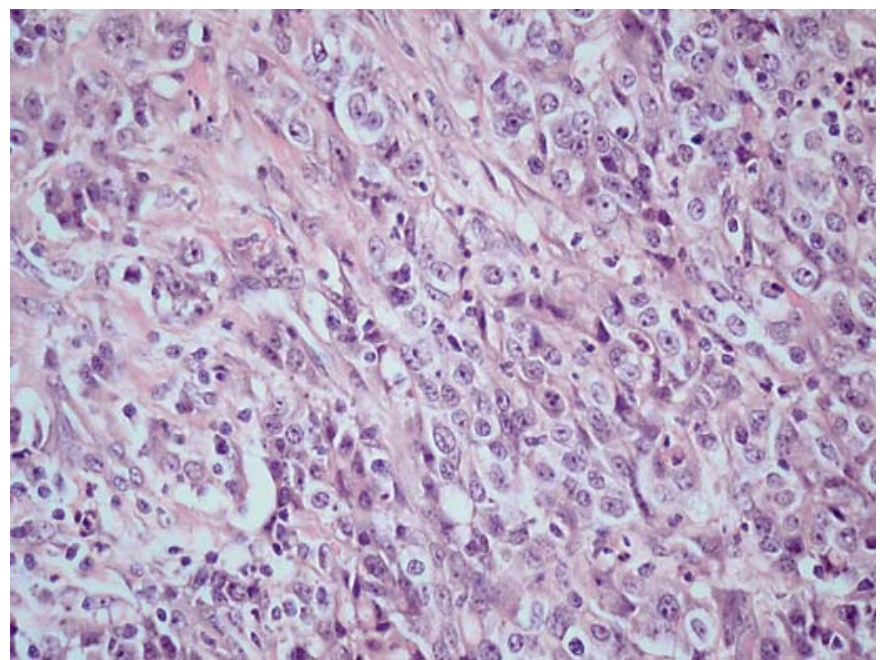

Fig. 2. Parotid metastasis. $\times 400$. HE.
-3 Moul JW, Paulson DF: Prostate cancer with solitary parotid metastasis correctly diagnosed with immunohistochemical stains. J Urol 1989; 142:1328-1329.

4 Mason AC, Azari KK, Farkas LM, Duvvuri U, Myers EN: Metastatic adenocarcinoma of the colon presenting as a mass in the mandible. Head Neck 2005;27:729-732.

5 Ozturk MA, Benekli M, Guler N, Altundag MK, Barsta I, Ayhan A: Gastric adenocarcinoma metastasis to the parotid gland. J. Clin Gastroenterol 1997;25:698-699.

6 Cantera JMG, Hernandez AV: Bilateral parotid metastasis as the initial presentation of a small cell lung carcinoma. J Oral Maxillofac Surg 1989;47:1199-1201.

7 Zachariades $\mathrm{N}$ : Neoplasms metastatic to the mouth, jaws and surrounding tissues. J Craniomaxillofac Surg 1989;17:283-290.

8 Batson OV: The function of the vertebral veins and their role in the spread of metastases. 1940. Clin Orthop Relat Res 1995;312:4-9.

>9 Willard CC, Weber CR, Buche W: Metastatic esophageal adenocarcinoma involving the maxilla. J Oral Maxillofac Surg 2002;60:14961499.

J.M. Schoneveld

Albert Schweitzer Hospital

PO Box 444

NL-3300 AK Dordrecht (The Netherlands)

Tel. +31 7865411 11, Fax +3178 6523569

E-Mail j.m.schoneveld@asz.nl 